\title{
Ulcerative colitis developing after amoebic dysentery in a haemophiliac patient with AIDS
}

\author{
I Sturgess, S M Greenfield, J Teare, M J O’Doherty
}

\begin{abstract}
Severe diarrhoea in patients with the acquired immune deficiency syndrome (AIDS) is usually a manifestation of gastrointestinal infection by a variety of organisms. We report a patient with low CD4 $\mathrm{T}$ cell counts who developed ulcerative colitis after amoebic dysentery. He subsequently developed acute ulcerative colitis with toxic dilatation while he was severely immunocompromised. He responded to corticosteroids and mesalazine and remains well on maintenance therapy.
\end{abstract}

Diarrhoeal illness is a common occurrence in patients with the acquired immune deficiency syndrome (AIDS). This is frequently the result of infection with opportunistic organisms, although diarrhoea without infection does occur as part of chronic human immunodeficiency virus (HIV) infection. We report a heterosexual man with haemophilia who presented with toxic megacolon secondary to ulcerative colitis and responded to steroids and mesalazine. To determine the extent of his colitis an "Indium oxine labelled leukocyte scan was performed, but in view of his neutropaenia donor leukocytes were used. To our knowledge, this is the first case of severe ulcerative colitis developing in an HIV infected patient with such severe immunosuppression.

\section{Case report}

A 44 year old heterosexual man with haemophilia who had had chronic HIV infection since 1984 presented to St Thomas's Hospital in February 1989 with diarrhoea. At that time Entamoeba histolytica was isolated and the diarrhoea responded to a course of tinidazole. There was a history of recent travel to Hong Kong, which accounted for his development of amoebic dysentery. He presented again in March 1989 with a further diarrhoeal illness and a weight loss of $3 \mathrm{~kg}$. On this occasion, although no pathogens were isolated from the stool, metronidazole was given at a dose of $400 \mathrm{mg}$ three times daily for 10 days. A sigmoidoscopy showed mild proctitis without ulceration and the rectal biopsy specimen showed a florid proctocolitis with crypt abscess formation Culture and immunofluorescence for cytomegalovirus were negative. At that time his CD4 counts were $0.22 \times 10^{\circ} / 1$ with a CD4/CD8 ratio of $0 \cdot 47$. In view of his diarrhoea and weight loss his disease was classified as Communicable Disease Centre stage $4 \mathrm{a} .{ }^{1} \mathrm{He}$ was begun on zidovudine (AZT) which resulted in improvement in his symptoms, with increased weight and resolution of his diarrhoea.

In late December 1989, he presented again with bloody diarrhoea. He was passing up to 10 fluid motions per day, and the diarrhoea had worsened progressively over the preceding three weeks despite a course of metronidazole two weeks before admission to hospital. The bleeding responded to repeated Factor VIII cover but his diarrhoea continued unabated. He had general malaise and anorexia and had lost $6 \mathrm{~kg}$ in weight. He was feverish, with temperatures up to $38.5^{\circ} \mathrm{C}$. There was generalised colicky abdominal pain and distension, particularly in the upper part of his abdomen. He was tender to palpation in the left iliac fossa and bowel sounds were increased.

Investigations at this time showed a haemoglobin concentration of $10 \cdot 1 \mathrm{~g} / 100 \mathrm{ml}$, a white cell count of $2 \cdot 6 \times 10^{9}$ (neutrophils $2 \cdot 1 \times$ $10^{9} / 1$, lymphocytes $0.4 \times 10^{9} / 1$, CD4 $0.17 \times 10^{9} / 1$, and a CD4/CD8 ratio of $0 \cdot 40$ ). Plain abdominal $x$ ray showed faecal loading of the right hemicolon but no gross dilatation. Repeated stool cultures and examination failed to identify any pathogens, particularly Mycobacterium tuberculosis, Cryptosporidium, Toxoplasma, Entamoeba, Microsporidium, Salmonella, and Shigella. No viruses were seen on electron microscopy nor was cytomegalovirus grown or identified using immunofluorescence from stools, urine, throat swab, or blood. Sigmoidoscopy at this time showed an acutely inflamed rectal mucosa with some contact bleeding. Biopsy specimens taken at sigmoidoscopy failed to show any evidence of

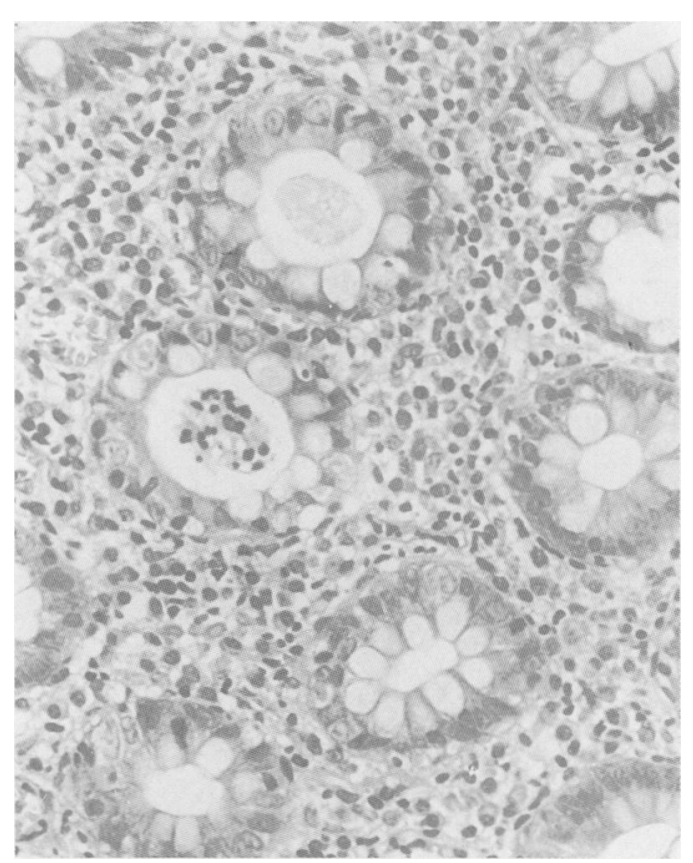

Figure 1: Rectal biopsy specimen showing marked acute inflammatory infiltrate of the lamina propria with extension into crypts and focal crypt abscess formation. (Original magnification $\times 400$. 


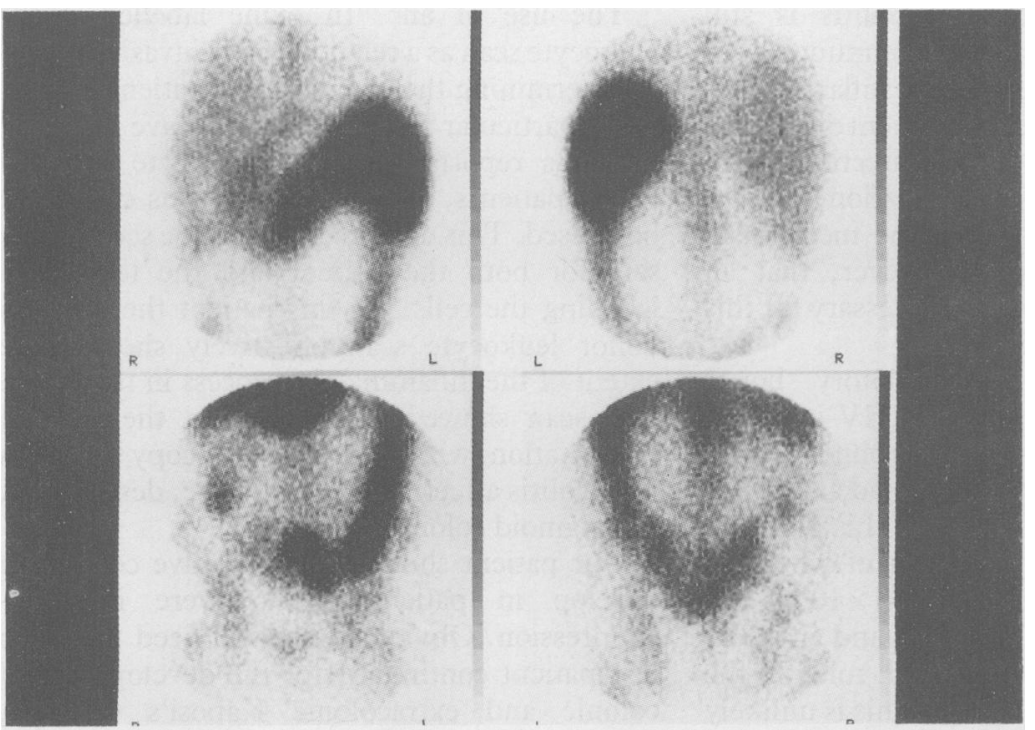

Figure 2: An "Indium labelled donor leukocyte scan showing a pancolitis. Reproduced with kind permission of Professor E11, Editor, European Journal Nuclear Medicine.

cytomegalovirus infection, and showed histologically an acute neutrophil infiltration with crypt abscess formation (Fig 1). No inclusion bodies were seen nor was Cryptosporidium identified. An "'In oxine labelled donor leukocyte scan (Fig 2) showed a pancolitis without abscess formation.

Because of his clinical state, intravenous gancyclovir was begun on the presumptive diagnosis of cytomegalovirus colitis while the above results were awaited. Over the next 48 hours his clinical state deteriorated with a rising temperature, tachycardia, and a reduction in the stool frequency. A plain abdominal film at this time showed toxic dilatation of the colon (Fig 3). Because of this and the repeatedly negative microbiological and virological studies, treatment with $100 \mathrm{mg}$ of intravenous hydrocortisone six hourly was begun. Broad spectrum antibiotics were also started. On this regimen his symptoms rapidly settled, with resolution of his fever and reduction in the dilatation of his colon on serial $x$ ray examination (Fig 3 ). Mesalazine, at a dose of $400 \mathrm{mg}$ three times daily, was begun 48 hours after the steroids. After a further 48 hours of this regimen the gancyclovir was stopped, without any deterioration in the patient's clinical state. Repeat studies for cytomegalovirus were again negative. Conversion to oral prednisolone, with a progressive reduction in dosage to $10 \mathrm{mg}$ daily, and an increase to $800 \mathrm{mg}$ three times daily of mesalazine over the ensuing two months has maintained remission. In this patient a clinical diagnosis of ulcerative colitis was guided by a poor response to antiviral treatment, positive histology, an "In labelled leukocyte scan compatible with ulcerative colitis, and the rapid onset of toxic megacolon.

Colonoscopy, performed two months after hospital discharge, showed a quiescent colitis from the distal transverse colon to the rectum. Multiple biopsy specimens taken at this examination did not show any evidence of cytomegalovirus colitis. There was evidence of a continuing, although mild, colitis and no histological evidence of Kaposi's sarcoma could be detected.

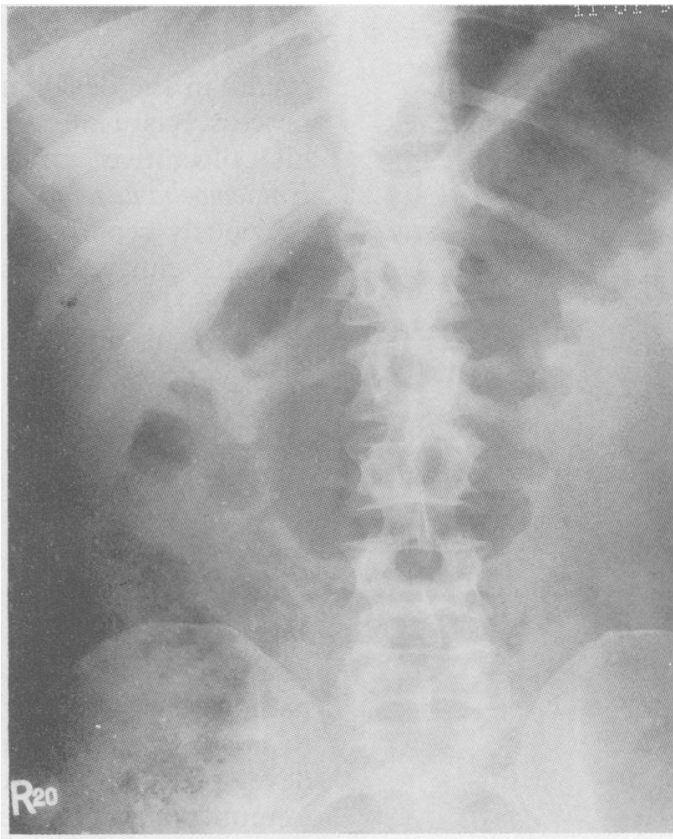

Figure $3 A$

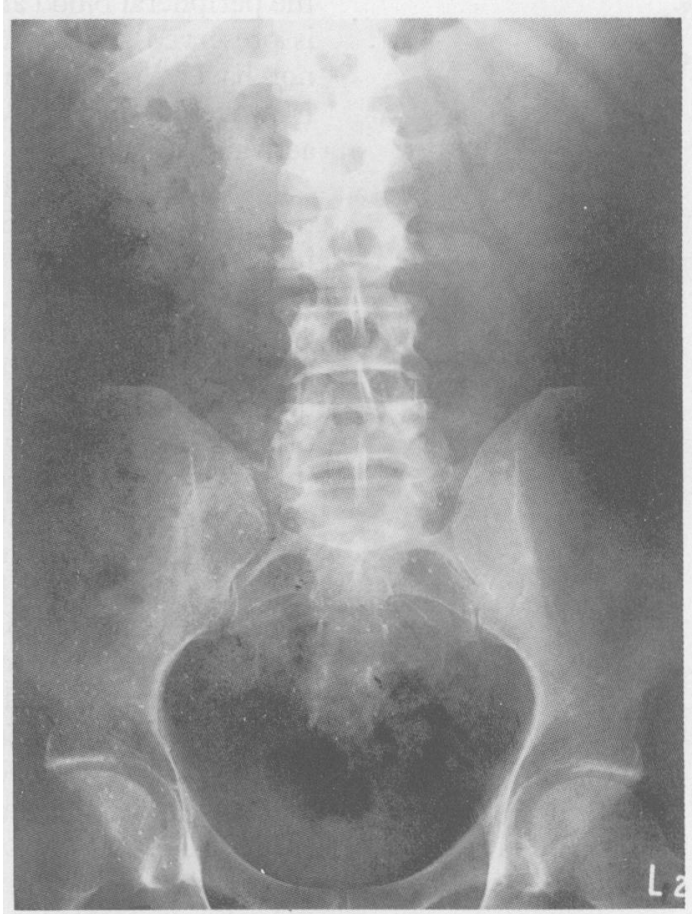

Figure $3 B$

Figure 3: (a) Plain abdominal $\mathrm{x}$ ray with toxic dilatation affecting the transverse colon. (b) Repeat abdominal film after five days of intravenous hydrocortisone showing resolution of the toxic dilatation.

There remains no evidence of cutaneous or oropharyngeal Kaposi's sarcoma.

\section{Discussion}

The occurrence of ulcerative colitis in a patient with AIDS presents a difficult diagnostic problem, particularly when the clinical state is rapidly deteriorating before the results of full microbiological investigations have been obtained. 
The aetiology of ulcerative colitis is still unknown, as are the initiating immunological events in the development of the inflammatory process. It is of interest that our patient developed his ulcerative colitis after infection with Entaemoeba histolytica. This association has been previously reported, ${ }^{2}$ although the mechanism remains elusive. It seems, however, that an intact CD4 $\mathrm{T}$ cell system is not necessary for this to initiate ulcerative colitis.

Previous reports of inflammatory bowel disease in patients with chronic HIV infection have included the remission of Crohn's disease with HIV infection. ${ }^{3}$ This patient had an 18 year history of recurrent relapses in his Crohn's colitis, his disease became quiescent when his CD4 $\mathrm{T}$ cell count dropped to $0.41 \times 10^{9} / 1$. The author suggested that CD4 $\mathrm{T}$ cells and an intact immune response play a central role in the pathogenesis of Crohn's disease. This is unlikely to be so in ulcerative colitis since our patient developed his disease while he was severely immunocomprised: his CD4 $\mathrm{T}$ cell count was $0 \cdot 22 \times 10^{9} / 1$. In addition $\mathrm{T}$ lymphocyte studies in ulcerative colitis have shown reductions in the overall numbers of both CD4 and CD8 cells in the peripheral blood and the ratio between them is preserved suggesting that $\mathrm{T}$ lymphocytes do not have a role in the inflammatory process. ${ }^{+}$ However, others have suggested a role for activated T cells. ${ }^{5}$

Ulcerative colitis has been reported once previously in a patient with chronic HIV infection, ${ }^{6}$ although this patient was not immunocompromised. The other reports of severe ulcerative colitis in homosexual AIDS patients showed that they had Kaposi's sarcoma of the bowel..$^{78}$ In both these cases an initial diagnosis of ulcerative colitis was made, but there was incomplete control of symptoms and in one the development of a toxic megacolon required colectomy resulting in the correct diagnosis being made. Both these patients developed cutaneous Kaposi's sarcoma soon after beginning steroids. In our patient, despite an exhaustive search, we have been unable to detect any evidence of Kaposi's sarcoma, although he will remain under colonoscopic surveillance. The resolution of symptoms with weight gain and continuing improved health three months after his episode of toxic dilatation suggest an episode of acute ulcerative colitis without any underlying Kaposi's sarcoma.
The use of an "'In oxine labelled donor leukocyte scan as a relatively non-invasive means of determining the extent of this patient's colitis is of particular interest. There have been no previous reports of donor leukocyte scans in AIDS patients, although autologous cells have been used. This donor cell technique seems to be safe for both the patient and the technician labelling the cells. ${ }^{9}$ In this patient the use of a donor leukocyte scan effectively showed the extent of the inflammatory process in the colon. The scan showed a pancolitis at the time of presentation, while a later colonoscopy showed a mild colitis affecting the transverse, descending, and sigmoid colon.

Our patient shows that ulcerative colitis can develop in patients with severe immunosuppression. Obviously, we will need to screen this patient continually for the development of colonic and extracolonic Kaposi's sarcoma, although previous reports have only shown this complication in homosexual AIDS patients. We would suggest that AIDS patients who develop ulcerative colitis should have colonoscopy and deep biopsy specimens taken six monthly or whenever there is a relapse. In this group of patients there is still the possibility that development of acute diarrhoea may be due to bacterial, viral, fungal, or protozoal infection as well as a relapse of ulcerative colitis.

In conclusion, this patient represents the first case of the development of ulcerative colitis with toxic dilatation in a patient with AIDS and adds another differential diagnosis to the list of causes of diarrhoea in these patients.

1 Centres for Disease Control. Classification system for human T lymphotropic virus type III/lymphadenopathy associated virus infections. MMWR 1986; 35: 334-9.

2 Rampton DS, Salmon PR, Clark CG. Nonspecific ulcerative colitis as a sequel to amebic dysentery. 7 Clin Gastroenterol 1983; 5: 217-9.

3 James SP. Remission of Crohn's disease after human immunodeficiency virus infection. Gastroenterology 1988; 95: 1667-9.

4 Selby WS, Jewell DP. T lymphocyte subsets in inflammatory bowel disease: peripheral blood. Gut 1983; 24: 99-105.

5 Trejdosiewicz LK, Badr-el-Din S, Smart CJ, et al. Colonic mucosal T lymphocytes in ulcerative colitis. Dig Dis Sci 1989;

6 Franke $M$, Kruis W, Heitz W. First manifestation of ulcerative colitis in a patient with HIV infection. Gastroenterology 1990; 98: 544-5.

7 Biggs BA, Crowe SM, Lucas CR, Ralston M, Thompson IL, Hardy KJ. AIDS related Kaposi's sarcoma presenting as ulcerative colitis and complicated by toxic megacolon. $G u$ 1987; 28: 1302-6.

8 Weber JN, Carmichael DJ, Boylston A, Munro A, Whitear WP, Pinching AJ. Kaposi's sarcoma of the bowel - presenting as apparent ulcerative colitis. Gut 1985; 26: 295-300.

9 O'Doherty MJ, Revell P, Page CJ, Lee S, Mountford PJ Nunan TO. Donor leucocyte imaging in patients with AIDS A preliminary report. Eur $\mathfrak{J}$ Nucl Med 1990; 17: 327-33. 